\title{
Design and Analysis of an Abbé Free Coplanar Stage
}

\author{
Tien-Tung Chung, Teng-Hui Tseng, and Ching-Siong Lim Chan \\ Dept. of Mechanical Engineering, National Taiwan University, Taipei 10617, Taiwan.
}

\begin{abstract}
Design and analysis of a new Abbé free coplanar xy stage are presented in this paper. The xy stage is formed as conventional xy stages which combine with two stacked up linear guides. The $\mathrm{x}$-guide is on the bottom and the y-guide is on top of the $x$-guide. The travel range of this xy stage is $300 \mathrm{~mm} \times 300 \mathrm{~mm}$, which fits dimensions of 12-inch wafers. A special mechanism is designed such that the z-surface of the y-guide has the same height as the zsurface of $\mathrm{x}$-guide, and the Abbé error and cumulative error of this coplanar stage can be reduced. The concept of symmetric structure design is also considered to eliminate the structural deformation due to driving force of two guides. For this long travel range precision stage, the finite element method (FEM) is applied to analyze the structural deformation and vibration natural frequencies. The requirement of structural deformation due to self-weight load is limited to $1.5 \mu \mathrm{m}$, and first natural frequency is limited to over $100 \mathrm{~Hz}$.
\end{abstract}

\section{Introduction}

Recently, precision positioning technique is important in the development of science and technology. The positioning stages with high accuracy is required in various fields such as semiconductor manufacturing, precision machining, precision measurements, etc. $\mathrm{XY}$ stages with long travel range are normally combined with two stacked up linear stages [1]. The traditional configuration of stacked up type stages has disadvantages of large assembly errors and geometry errors, such as Abbé error. Therefore, stacked up type stages reduce the motion stability and accuracy for positioning. This paper proposes a design of the coplanar XY stage to reduce the accumulation of geometric errors and Abbé error. The coplanar stage developed in this paper contains several advanced design methods, including Abbé free concept and coplanar concept.

\section{Coplanar XY stage design}

Linear encoder is usually used to measure displacement in direct drive linear stage. If the work piece is put on the moving stage, the work piece and the linear encoder are on the different z-plane [2]. When the moving stage is unstable, the Abbé offset will appear, as shown in Fig. 1. To eliminate the Abbe offset in the direct driver linear stage, laser interferometer is considered, as shown in Fig.2. Reflection mirror, moving stage and laser interferometer locate at the same height. The laser beam will be emitted from the laser interferometer to the reflection mirror and will be reflected back into the receptacle on the laser interferometer, the displacement of the work piece will be measured by the laser interferometer. The laser interferometric measurement is free from Abbé errors of the first order in all the three xyz coordinates [3].

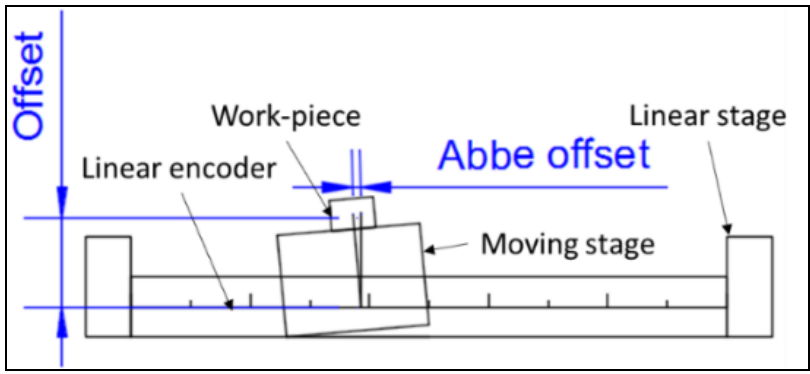

Figure 1. Abbé offset in a direct driver linear stage

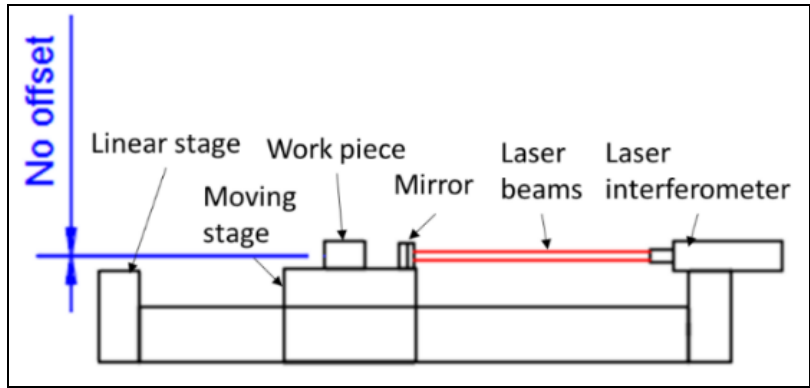

Figure 2. Elimination of abbé offset

Conventional $\mathrm{XY}$ stage is stacked up by two linear guides. The Abbé error of the lower guide is high because of the vertical distance from work piece to the bottom of the stage. The concept of coplanar stage is proposed to eliminate the Abbé error. As shown in Fig. 3, point 1 is $\mathrm{X}$-direction laser interferometer, point 2 is $\mathrm{X}$-direction reflection mirror, point 3 is Y-direction laser interferometer and point 4 is $\mathrm{Y}$-direction reflection mirror. 
The Abbé error in a two-dimension space can be eliminated when the point 1 , point 2 , point 3 , and point 4 are of the same z-plane heights and the line of point 1 and point 2 intersect the line of point 3 and point 4 .

The coplanar stage is proposed in this paper, as shown in Fig. 4. The Y-plate is supported by two precision guideways that are mounted on the X-plate. The X-plate is supported by another two precision guideways that are mounted on the granite. According to the coplanar design and Abbé free design, component 1, component 2, component 3 , component 4 , component 5 , and component 6 have the same z-plane heights. Component 1, component 2 and component 3 are in one straight line in $\mathrm{X}$-direction. Component 4, component 5 and component 6 are in one straight line in $\mathrm{Y}$-direction. The line in $\mathrm{X}$ direction intersects the line in $\mathrm{Y}$-direction, located at the measurement point on the surface of the work piece to be measured [4]. This is a combined concept of coplanar design and Abbé free design.

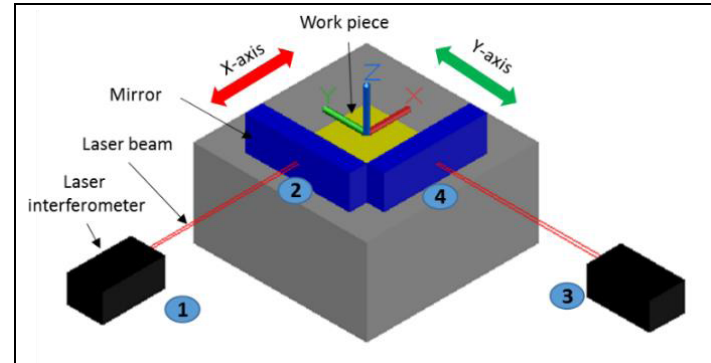

Figure 3. Illustration of an Abbé free design coplanar stage

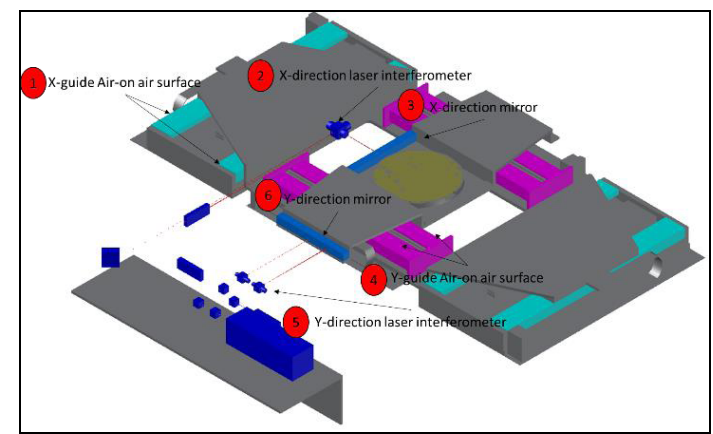

Figuer 4. Coplanar stage and laser measuring system

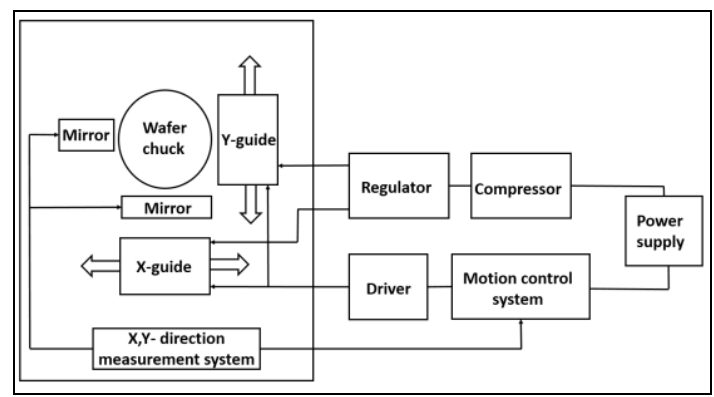

Figure 5. System profile of coplanar motion stage

The overall system configuration of the coplanar motion stage is shown in Fig. 5. The coplanar stage is controlled by the motion control system through the driver connected to a computer. This positioning system is designed for wafer inspection. The exploded view of the coplanar stage is shown in Fig. 6. The Y-stage incorporates a wafer chuck, two mirrors, a Y-plate, and two Y-guides, as shown in Fig. 7. The X-stage incorporates X-plate, two air bearings, and two X-guides, as shown in Fig. 8. Fig. 9 shows the box-in-box concept, which makes the guideways in $\mathrm{X}$-direction and $\mathrm{Y}$ direction are of the same height.As a result, the Abbé error and the cumulative error can be reduced [5] .

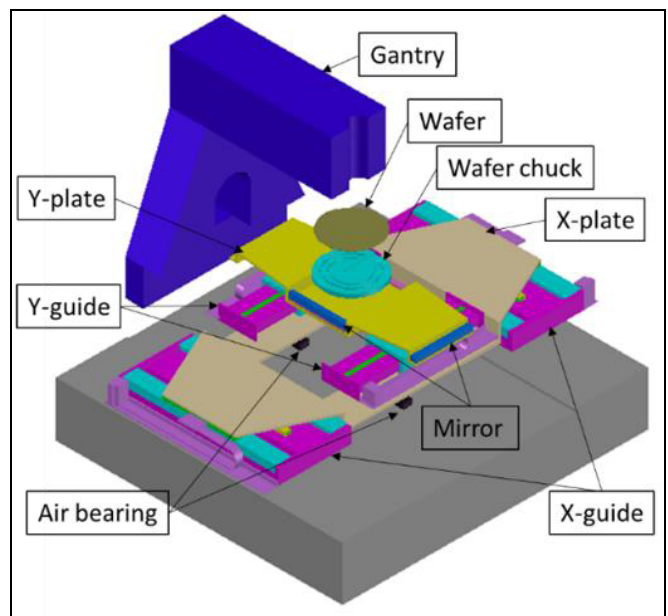

Figure 6. Exploded view of the positioning stage

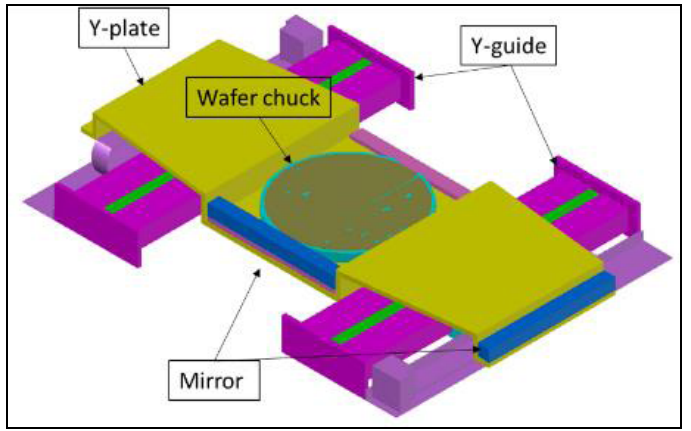

Figure 7. Isometric view of Y-stage

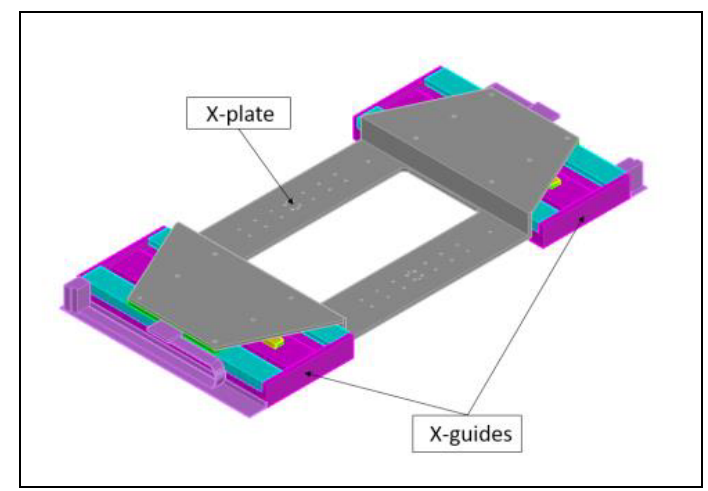

Figure 8. Isometric view of $\mathrm{X}$-stage

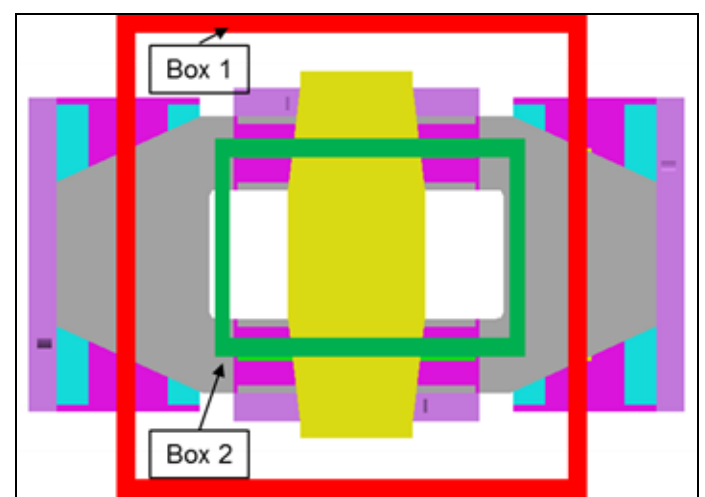

Figure 9. Box-in-box design 


\section{Finite element analysis}

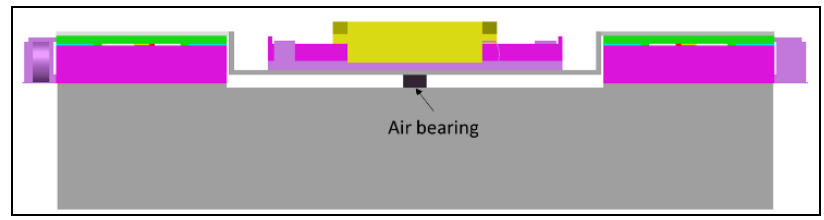

Figure 10. The front view of $\mathrm{X}$ - and $\mathrm{Y}$-stage

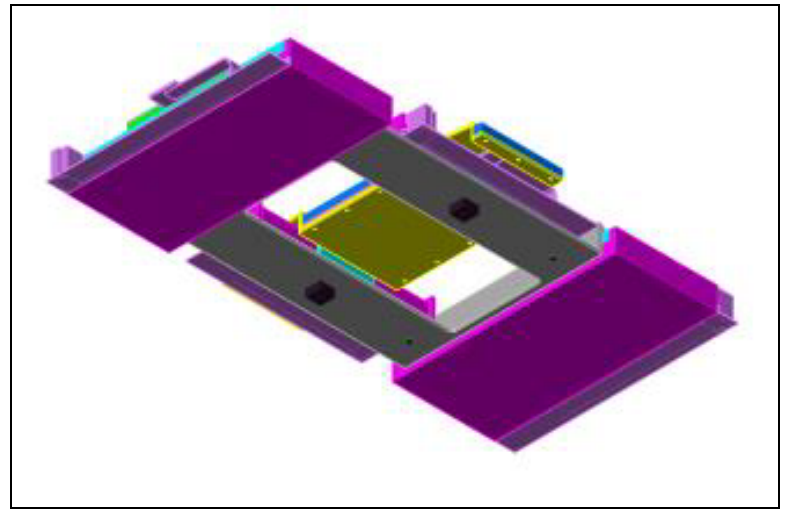

Figure 11. The isometric view of $\mathrm{X}$ - and $\mathrm{Y}$-stage

Structure deformation is important in this coplanar stage. Positioning accuracy will be influenced when the deformation is too large. To reduce self-weight deformation, two air bearings are mounted under the $\mathrm{X}$ stage, as shown in Fig. 10. Isometric view of $\mathrm{X}$ - and $\mathrm{Y}$ stage with two air bearings are shown in Fig. 11. Then the self-weight deformation of the X-and Y- stages can be analyzed by using finite element analysis software ANSYS. The deformation of the $\mathrm{X}$ - and Y- stages is
$1.469 \mu \mathrm{m}$, as shown in Fig. 12. The deformation of the gantry is $1.459 \mu \mathrm{m}$, as shown in Fig. 13.

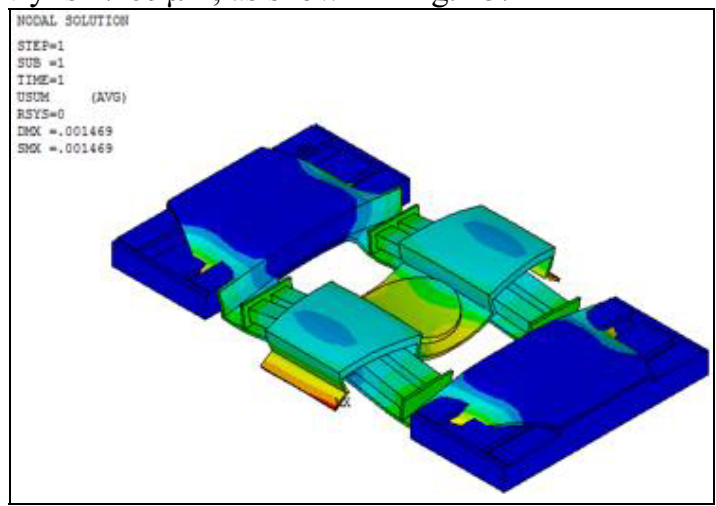

Figure 12. Maximum deformations of $\mathrm{X}$ - and $\mathrm{Y}$ - stages

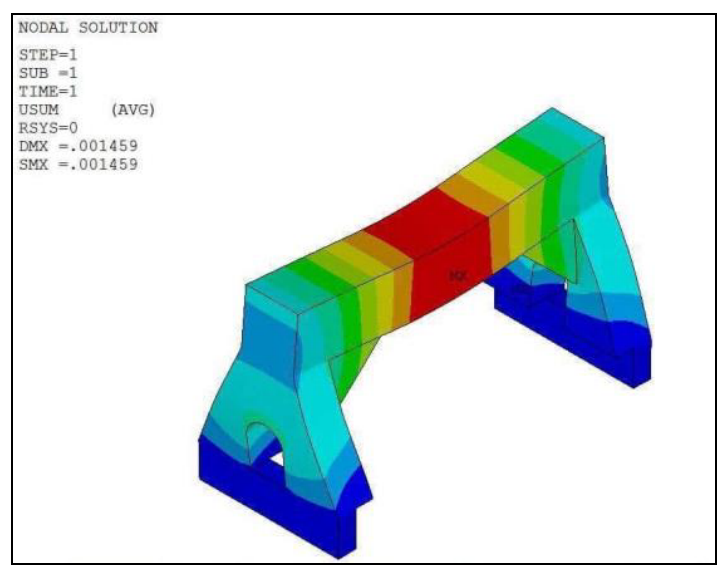

Figure 13. Maximum deformation of gantry

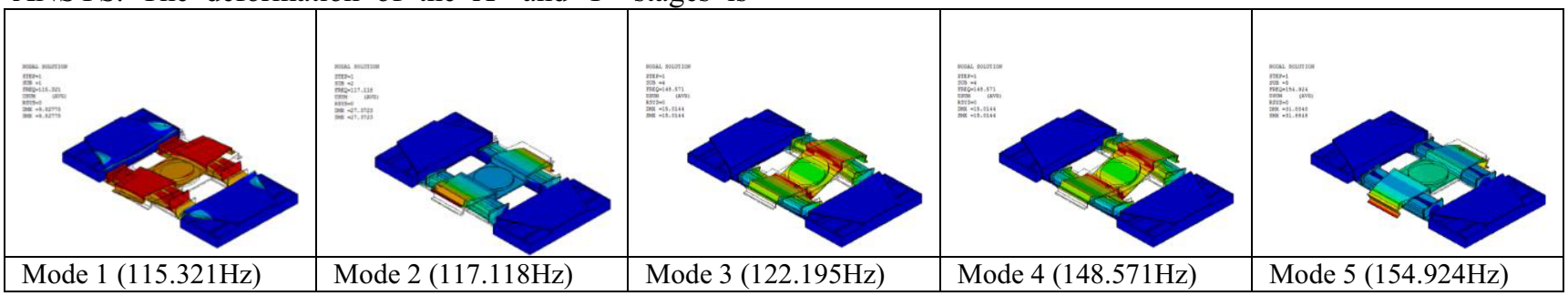

Figure 14. Mode shapes and natural frequencies of $\mathrm{X}$-and Y-plate

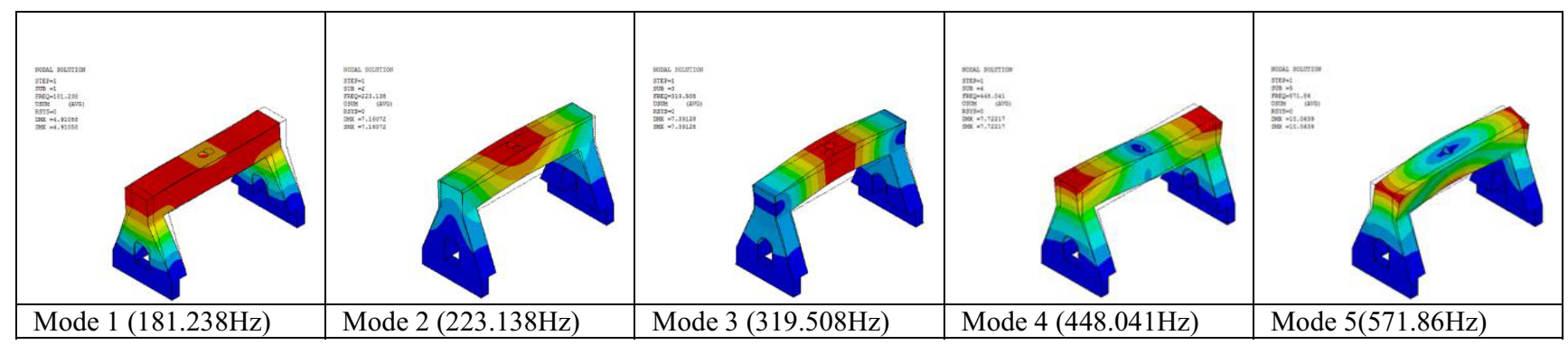

Figure 15. Mode shapes and natural frequencies of gantry

The design of the high accuracy positioning stage needs to consider not only the structure deformation but also the natural frequency of the stage structure. The coplanar stage must have the operating frequency far from its structure natural frequency in order to prevent the resonance of the structure, which will influence the accuracy of the stage seriously. Hence, it is necessary to analyze the natural frequency and mode shape of coplanar stages and gantry. For a high accuracy requirement, the operating frequency of the controlling system should be different from the structure operating frequency.

The results of the first five modes and mode shapes from 1 st to 5 th of X- and Y- stages are shown in Fig. 14. The 1 th mode is a translation motion in X-direction, the 2 th mode is rotation motion in $\mathrm{X}$-direction, the 3 th mode is translation motion in Z-direction, the 4th mode is rotation motion about $\mathrm{X}$-direction, and the 5 th mode is 
translation motion about Z-direction. From the Fig. 14, the frequencies of first five modes are between $100 \mathrm{~Hz}$ and $160 \mathrm{~Hz}$. The natural frequency will be changed due to different mechanism design. To avoid resonance, the mechanism design must be altered as the frequencies of the coplanar motion stage are measured.

\section{Summary}

This paper proposed a new coplanar XY stage used for wafer inspection. This coplanar stage with special mechanism design is Abbé free in both $\mathrm{X}$ - and $\mathrm{Y}$ directions. The travel range of this coplanar motion stage is $300 \mathrm{~mm} \times 300 \mathrm{~mm}$, which makes it possible for a 12 inch wafer inspection. The self-weight analysis and modal analysis are carried out by using finite element analysis software ANSYS. The structural characteristic of this coplanar stage satisfies the design requirements of given design specifications.
The results of the first five modes and mode shape from 1 st to 5 th of gantry are shown in Fig. 15. The 1st mode is a translation motion in $\mathrm{Y}$-direction, the 2 nd mode is translation motion in $\mathrm{X}$-direction, the $3 \mathrm{rd}$ mode is translation motion in Z-direction, the 4 th mode is rotation motion about $\mathrm{Z}$-direction, and the 5 th mode is rotation motion about X-direction.

\section{References}

1. Information on http://www.hiwin.com/pdf/linear motors.pdf.

2. Osita D. I. Nwokah, and Yildirim Hurmuzlu, CRC press LLC, pp.160-162, (2001)

3. Gunter Wilkening and Ludger Koenders, Nanoscale Calibration Standards and Methods: Dimensional and Related Measurements in the Micro- and Nanometer Range, Wiley-VCH, pp. 47-59, (2005).

4. Bryan, J.B., Precision Engineering, pp.129-132,(1979).

5. W. L. Wang, Y. T. Fei, and K. C. Fan, Proceedings of the 1 st IEEE International Conference on Nano/Micro Engineered and Molecular Systems, Zhuhai, China, pp. 18-21, (2006). 